\title{
Current Trends in Robot-Assisted Upper-Limb Stroke Rehabilitation: Promoting Patient Engagement in Therapy
}

\author{
Amy A. Blank • James A. French • Ali Utku Pehlivan • \\ Marcia K. O'Malley
}

Published online: 28 June 2014

(C) Springer Science + Business Media New York 2014

\begin{abstract}
Stroke is one of the leading causes of long-term disability today; therefore, many research efforts are focused on designing maximally effective and efficient treatment methods. In particular, robotic stroke rehabilitation has received significant attention for upper-limb therapy due to its ability to provide high-intensity repetitive movement therapy with less effort than would be required for traditional methods. Recent research has focused on increasing patient engagement in therapy, which has been shown to be important for inducing neural plasticity to facilitate recovery. Robotic therapy devices enable unique methods for promoting patient engagement by providing assistance only as needed and by detecting patient movement intent to drive to the device. Use of these methods has demonstrated improvements in functional outcomes, but careful comparisons between methods remain to be done. Future work should include controlled clinical trials and comparisons of effectiveness of different methods for patients with different abilities and needs in order to inform future development of patient-specific therapeutic protocols.
\end{abstract}

A. A. Blank · J. A. French - A. U. Pehlivan .

M. K. O'Malley $(\square)$

Department of Mechanical Engineering, Rice University,

Houston, TX, USA

e-mail: omalleym@rice.edu

A. A. Blank

e-mail: amyblank@ rice.edu

J. A. French

e-mail: jaf12@rice.edu

A. U. Pehlivan

e-mail: aup1@rice.edu
Keywords Robotic stroke rehabilitation · Upper-limb rehabilitation · Patient engagement · Assist-as-needed . Intent to move

\section{Introduction}

Stroke is one of the leading causes of long-term disability today, currently affecting 6.8 million people in the United States alone, and this count is expected to rise to over 10 million people by the year 2030. As of 2010, estimated costs for treatment and care for stroke survivors totaled over $\$ 20$ billion [1]. To restore stroke survivors' independence and reduce the cost of therapy and care, recent research in stroke rehabilitation has emphasized the need for more effective therapy than the current standard of care. In particular, more effective rehabilitation of arm function is crucial because of the limitations stroke survivors experience in performing activities of daily living [2].

There is evidence that intensive motion training including many repetitions can improve therapeutic outcomes both during the acute phase immediately after the stroke and in the longer term [3,4]. Because such intensive training can be costly and require significant effort, robotic rehabilitation systems have been proposed to aid the therapist in providing consistent, repeatable training with less effort [5-9]. Many of these systems have shown therapy outcomes comparable to equivalent intensive training without robotic aid $[10,11]$ or an equal number of unassisted movements $[12,13]$, suggesting that one of the primary benefits of robotic training is its ability to provide intensive therapy at lower cost and effort $[14,15]$.

There is also evidence that passive movements are insufficient to alter motor recovery [16], and that patients must be actively engaged and attempting to move $[17,18]$ 
in order to acquire the beneficial effects of robotic rehabilitation. Indeed, a patient-responsive protocol that progressively adapts robotic training and assistance based on measures of movement coordination to continually challenge patients has been shown to provide substantially better outcomes than previously reported for robotic therapy $[19,20]$. This need for active engagement in robotic therapy is consistent with evidence that active engagement induces neural plasticity in motor learning [21], suggesting that robotic therapy systems could be used to monitor patient intent and further promote active patient participation during therapy. Recent research has focused on promoting patient engagement by providing adaptive robotic training protocols $[22 \bullet, 23 \bullet, 24 \bullet, 25]$ and detecting and responding to patient movement intent $[19,26,27$, $28 \bullet$ ] to promote neural plasticity and optimize the effect of the therapy, thereby improving patient outcomes and reducing the cost of therapy. In this article, we will discuss these emerging trends in promoting patient engagement in therapy and directions for the future of the field.

\section{Development of Robotic Devices for Upper-Limb Stroke Rehabilitation: A Historical Perspective}

Assistive robotics to aid people with disabilities include a wide variety of systems, ranging from purely assistive devices such as robotic wheelchairs and robotic arms designed to assist users during activities of daily living to therapeutic devices designed to improve a user's ability to function independently [29]. In the area of therapeutic devices, robotic delivery of stroke therapy has been a topic of research for over two decades [30-32]. In upper-limb rehabilitation, the early systems used robotic manipulators to guide patient's hand and arm to the desired positions in a horizontal plane [33, 34]. These devices focused on rehabilitation of elbow and shoulder movement, typically immobilizing the patient's wrist to ensure that the desired motions arm were produced. Controller designs emphasized the safety of the robotic devices, using control methods specifically designed to ensure safe interaction forces between the user and the device [33]. The early clinical trials showed that robotic rehabilitation devices for the upper-limb were safe for human use and provided treatment benefits when used in addition to standard therapy $[33,35,36]$.

Later devices aimed to expand the capabilities of robotic therapeutic devices by targeting the more distal segments of the upper-limb, with wrist and hand modules to attach to previously developed arm devices [9, 37-39], standalone wrist and/or hand devices [6, 40-43], or whole limb devices that target the shoulder, elbow, and wrist joints simultaneously [44-46]. Many of these are exoskeleton type devices, which aim to isolate the motion of individual joints. These devices tend to have higher complexity and reduced range of motion as compared to endpoint manipulators, but they more effectively target the desired joint(s) by eliminating compensatory behaviors, and they enable more precise data collection about the motion of the patient's limb.

In parallel with the development of more capable robotic devices, clinical trials focused on identifying potential advantages of robotic therapy for upper-limb rehabilitation, including the benefits of active patient participation in therapy. Whereas early studies used robotic training as an added treatment in conjunction with standard therapy, later studies conducted more careful comparisons in which treatment groups with and without robotic assistance received equal amounts of movement therapy in an attempt to determine which specific aspects of robotic therapy resulted in functional improvements. In a clinical trial with 127 subjects, Lo et al. [47] compared functional outcomes for groups receiving three different therapy types: robotic therapy $(n=49)$; intensive therapy with the same movement intensity as the robotic therapy $(n=50)$; and standard therapy, which includes fewer repetitions than the intensive therapy $(n=28)$. In this study, robotic therapy provided similar functional outcomes to the intensive therapy, which consisted of the same amount of training, but without a robot. Both the robotic therapy and the intensive training provided improved functional outcomes when compared to the standard therapy. These results suggest that the primary advantages of current devices lie in the reduced cost and effort to provide the same training, especially because the intensive training regimen used in this study was not practical for a therapist to use as standard care [15]. Currently, the costs of these devices are comparable to the cost of intensive therapy [15], but future devices may become cheaper and more portable.

Another significant research focus has been the importance of patient engagement in therapy. In a randomized controlled trial with 32 stroke patients, Lynch et al. [16] found that continuous passive movement did not lead to improvements in motor function, indicating that although this type of therapy has shown possible benefits in neural recovery, it is not sufficient to produce measurable functional benefits. However, continuous passive movement coupled with active movement can contribute to motor recovery, as shown by numerous studies with robotic therapy devices [17-20, 48]. Other studies have found evidence that movement frequency and patient engagement are primary factors in improving functional outcomes, rather than the use of the robot itself $[12,49]$. These results agree with evidence that motor rehabilitation is a form of learning $[21,50,51]$, so engaging the correct areas of the brain through active patient participation should improve 
learning [21]. Thus, these studies point to a need to explore strategies for optimizing therapy protocols by promoting patient engagement in therapy.

\section{Toward Increased Patient Engagement in Therapy}

Since the importance of patient engagement has been demonstrated in many studies of robot-assisted stroke therapy $[12,17-20,48,49]$, recent work has focused on methods to engage patients more actively in their therapy. In the field of robot-assisted stroke therapy, there have been three main approaches to promoting patient engagement: (1) assist-as-needed algorithms to provide the minimal robotic assistance necessary for the patient to complete a motion, (2) detection of patient intent to move, and (3) virtual reality games for a more immersive experience. This review will focus on the first two approaches, which are more closely related to robotic systems. Both of these methods require effort from the patient to complete movements, but the nature of the effort and the timing is different, making these two schemes appropriate for different scenarios, depending on the needs of the patient. The virtual reality literature has been extensively reviewed elsewhere [52].

\section{Assist-as-Needed Control Algorithms}

Assist-as-needed algorithms focus on providing the minimal amount of robotic assistance necessary for a patient to complete a movement, thus requiring significant effort from the patient. Many methods have been proposed to determine the necessary amount of assistance (see Table 1). The simplest controllers of this type are impedance controllers designed to keep the patient's hand on a particular path during reaching motion. A simple impedance controller compares the position and velocity of the robot to a desired reference trajectory and applies a force proportional to a weighted sum of the position and velocity errors [66]. In the case of robotic stroke therapy, if the patient's hand deviates from the path, then these controllers provide a restoring force proportional to the perpendicular distance from the path $[55,56]$. Thus, if the patient is able to complete the prescribed movement successfully, then the robot does not apply any forces. Other controllers specify both a desired path and a desired completion time, adding assistance in moving along the path toward the target. A desired trajectory is defined-typically a minimum jerk trajectory (a specific trajectory type with a smooth, bellshaped velocity profile that minimizes the change in acceleration during the movement [67]) or an average trajectory pre-recorded from healthy subjects. Then, the robot provides an assistive force proportional to the distance the subject's arm lags behind the desired trajectory $[19,54]$. Although most of these controllers prescribe a specific trajectory between the starting point and the target point, there is also an alternate strategy of allowing subjects to choose their own trajectory, while still assisting movement toward the target [53]. Feasibility of these systems has been confirmed [53,54], and trials with stroke patients have demonstrated that these algorithms result in a reduction in muscle tone [19], significant functional improvements [68], and better functional improvements than robotic therapy designed to mimic the intensity of traditional therapy [17].

Although these controllers showed promise in clinical trials, there is still a tendency (often termed "slacking") for users to rely heavily on the assistive force [69]. To compensate for this tendency, many implementations include a forgetting term in the controller, which decreases the amount of assistance after each successful trial, encouraging more active participation by the subject $[22 \cdot$, $23 \bullet, 24 \bullet, 25,57,58 \bullet, 59-63,70-72]$. Many of these controllers explicitly model and estimate the inertia and damping of the robotic system and the patient's arm, and they typically compensate fully for forces due to these properties $[25,57,58 \cdot, 59,70,71,73]$. Many of them also model the patient's effort contribution at various points in the workspace (with or without inertia and damping compensation). Since an explicit model is not known, an estimate of the patient's effort is modeled using radial basis functions (RBFs) learned at many points covering the workspace, and these estimated functions are updated throughout training [22•, 25, 57]. Others use Bayesian learning techniques to determine the appropriate amount of assistance needed to complete the task [72]. In one implementation by Pérez-Rodríguez et al. [23•], the learned model of patient ability is used to anticipate deviations from a desired trajectory and apply corrective forces before the deviations occur, thus reducing the force applied by the robot. Wolbrecht et al. [25, 57] compared their adaptive controller with a forgetting term to a version without a forgetting term in trials with stroke patients and healthy subjects, showing that the forgetting term leads to higher levels patient involvement in therapy. More recent improvements in the model of patient effort have included directionality of movement as well, since stroke patients often have more difficulty with flexion than extension, or vice versa, but so far, this improved controller has only been validated with unimpaired subjects [65••]. This group also plans to include velocity dependence in their model in the future, and testing with stroke patients is planned $[65 \bullet \cdot]$.

Another promising alternative is to adaptively adjust the difficulty of the movement task. Masia et al. [64] asked subjects to track a target moving in a sinusoidal pattern 


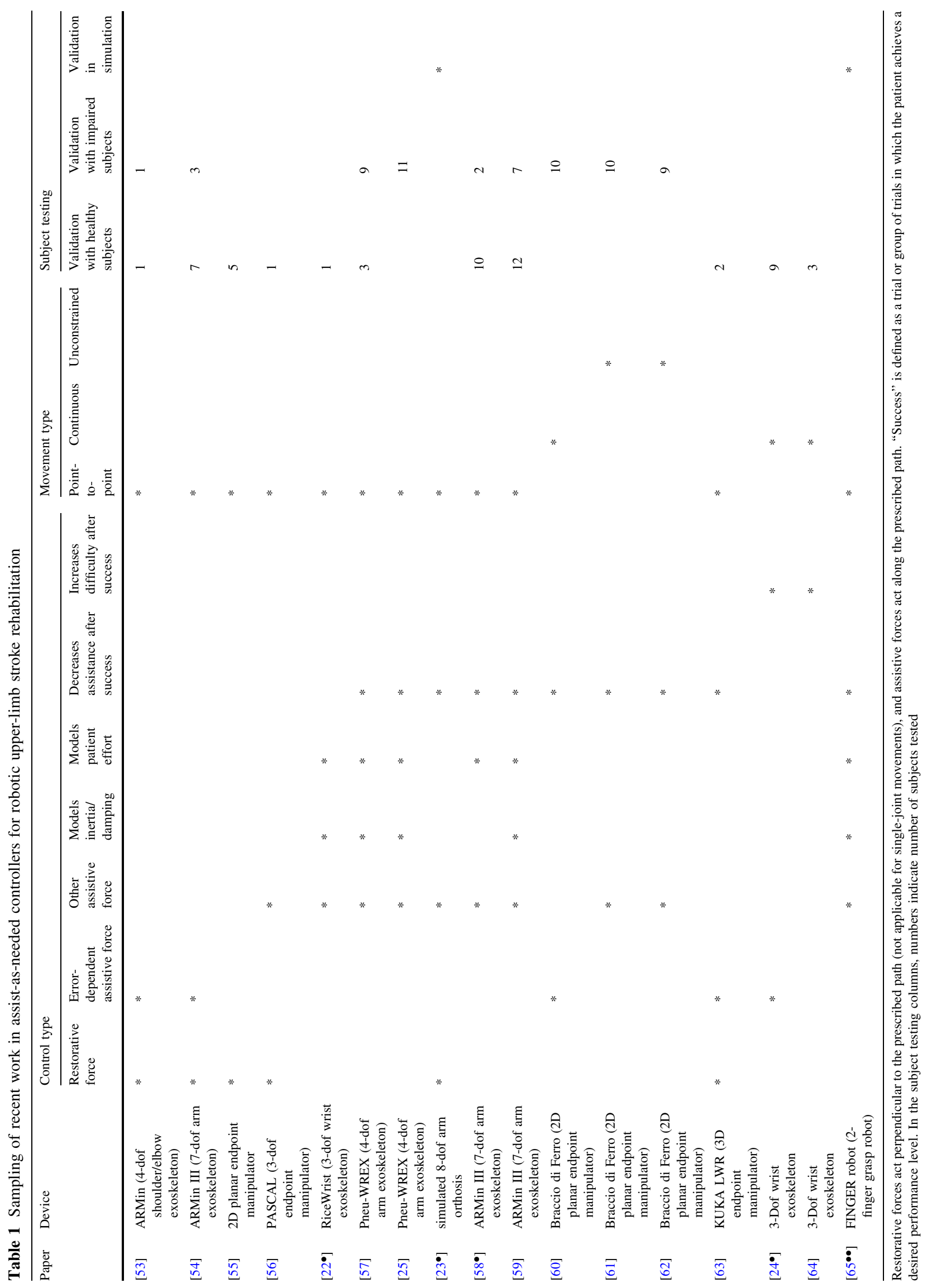


with wrist movement, and increased the required range of motion after each successful trial. Their preliminary results with three stroke subjects showed the potential of this method to increase subjects' range of wrist motion. A larger follow-up study by Squeri et al. [24•] with eleven stroke subjects further supported these results, showing improvements in the subjects' active range of motion and measurable improvements to motor function. A different approach to adjusting task difficulty was taken by Badesa et al. [74], who used machine learning methods to classify the subject's physiological state as "relaxed," "mediumstressed," or "over-stressed" based on a number of physiological measures (pulse rate, respiration rate, skin temperature, and galvanic skin response) and adjusted the task difficulty accordingly. Their results have shown feasibility of the proposed method, but patient trials have not yet been conducted.

Great promise has been shown for these assist-as-needed methods to encourage active patient participation in therapy by providing the minimum assistance necessary for the patient to complete a movement. Clinical trials thus far have demonstrated feasibility of the methods and functional improvements as a result of the training. However, controlled clinical trials are still needed to compare training methods and quantify possible benefits over traditional therapy.

\section{Detection of Patient Intent}

Detection of patient intent has also shown promise as a way to engage patients more actively in their therapy. Current methods include triggering based on force, velocity, or time thresholds, or detecting movement intent based on electromyography (EMG) or electroencephalography (EEG) (see Table 2). Particularly for severely impaired patients who have trouble completing movements, these approaches might help encourage patients to make an effort to move the affected limb. The simplest implementations of triggered assistance use force, position, or time cues as triggers. In force-triggered scenarios, robot assistance is initiated when the patient exerts force above a certain threshold in the movement direction [12, 38, 40, 49, 75]. A force trigger is appropriate for patients who have a severely limited range of motion but are able to exert some force near the starting position of the limb; in this case, the patient's movement intention causes the beginning of the movement, and the limb is carried through a range of motion that the patient would be unable to achieve unassisted. Similarly, a velocity trigger is appropriate for a patient who has a limited range of motion but is able to initiate movement near the starting position. Krebs et al. [19] implemented a velocity trigger in which the required velocity threshold decreased over time, in order to allow patients with different movement capabilities to use the device. In more extreme cases in which a patient is unable to produce sufficient force or velocity to trigger robotic assistance, a time trigger can also be used, such that the robot will begin to move the patient's passive limb after a certain amount of time has passed [86]. In this case, it is assumed that the patient is attempting to move the affected limb but is unable to do so. Another alternative for patients who are unable to produce significant force or velocity is to use gaze tracking to select a target and initiate motion [76•].

Other implementations rely on EMG signals from surface electrodes on the patient's arm to trigger-assisted movement upon detection of muscle activation over a specified threshold, since some patients may be able to produce muscle activity but not movement or force [19, 77, 79, 87-89]. These triggered approaches are useful for patients who have limited movement capability, but since they allow the patient to remain passive after triggering the movement, the patient's movement intention might not continue for the duration of the movement. Therefore, some groups have used EMG continuously throughout a movement to ensure continued patient engagement. For example, Song et al. [78•] provided an assistive force proportional to the measured EMG levels; their study with sixteen stroke subjects showed that this method helped subjects achieve a larger range of motion and improved targeting accuracy. However, continuous EMG control methods typically allow significant freedom of movement, rather than enforcing a normative movement profile; thus, a criticism of these methods is that they may reinforce pathological movement instead of encouraging recovery of normal movement patterns. Despite this drawback, the use of EMG signals to drive a robotic therapy device has been shown to generate improved muscle coordination and reduced spasticity in stroke patients [27, 87, 90], but these studies did not compare different treatment methods.

As another alternative, some researchers have advocated the use of EEG to detect movement intention through noninvasive surface electrodes on the patient's scalp [91]. One of the primary advantages of using EEG over EMG is that many patients have a tendency toward muscle spasms or activation of the wrong groups of muscles; using EEG allows the system to measure movement intention at the brain, thus bypassing these abnormal muscle activation patterns. EEG also allows participation by patients who cannot actually produce voluntary motion of the affected limb. In this case, mental practice of the movement can still activate the necessary regions of the brain, thus inducing some neural plasticity even if movement is not produced [92]. Since mental practice has been shown to be more efficient when combined with appropriate somatosensory 


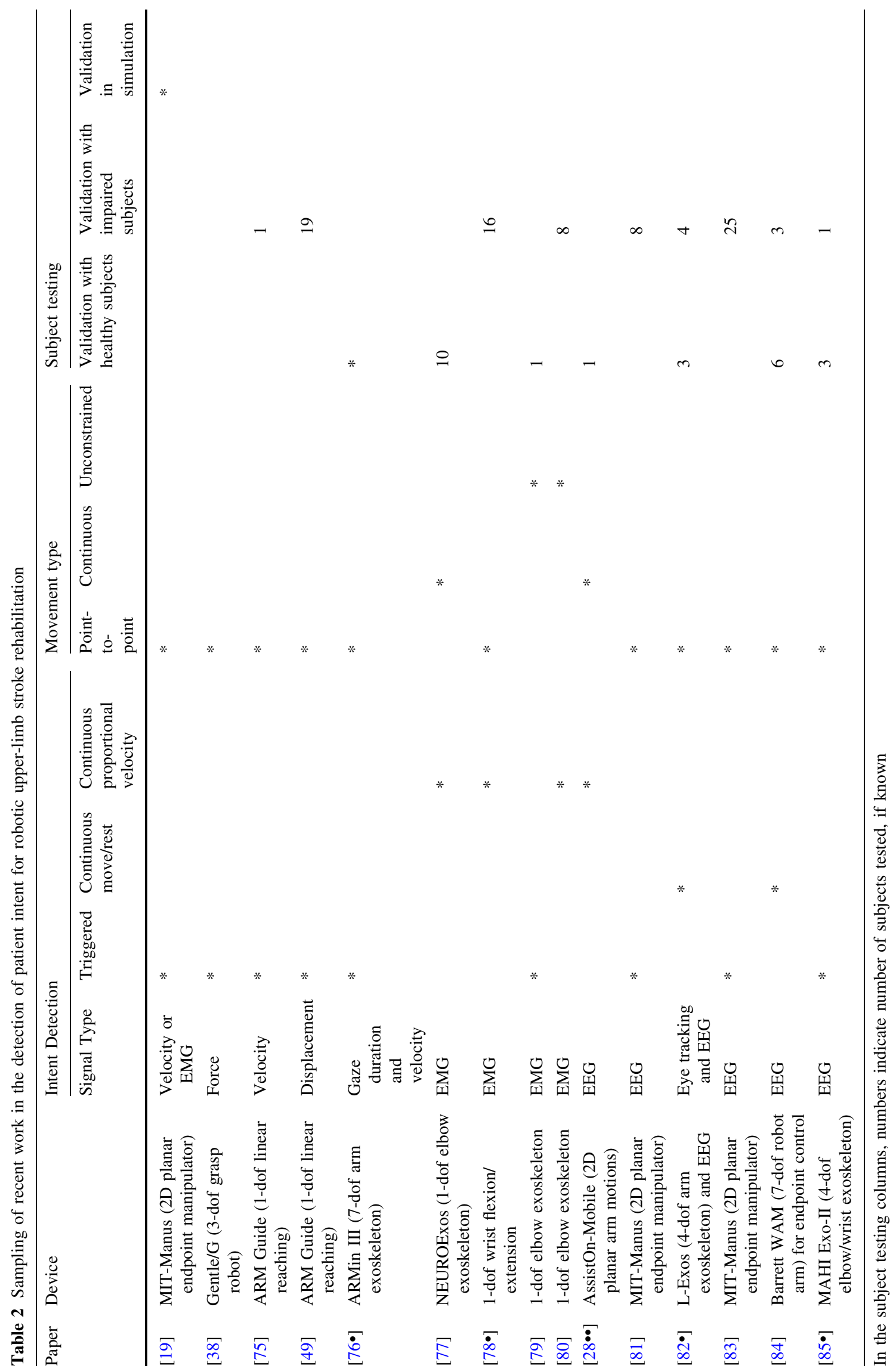


feedback [93], using a robotic system to move the patient's arm can improve the effectiveness of the training. Currently, most EEG-based systems use motor imagery to trigger movement using a two-state classification; subjects are asked to imagine the arm moving or at rest, and the resulting signals are classified as a "move" or "rest" cue to determine when to initiate robot assistance [81, 83-85•, 94, 95]. Typically, the movement is to a predefined target, though Frisoli et al. [82•] have enabled subjects to choose target objects independently by combining the EEG cue with eye tracking and a Kinect system to detect when the subject is focusing attention on an object in the environment. More recently, continuous control has been demonstrated using the move/rest classification probability to command a movement speed in the robotic system [29••]. EEG-triggered systems have been shown to be feasible and able to detect movement intention with high accuracy [29, $81,82 \cdot, 84]$, and in some cases, they have been shown to compare favorably to standard robotic therapy [95], but large-scale controlled clinical trials remain to be done.

\section{The MAHI EXO-II: A Case Study in Adaptive Robotic Training for Wrist Rehabilitation}

As an example of a system with an adaptive assist-asneeded controller and detection of patient intent, we consider the MAHI EXO-II and the RiceWrist, two versions of a wrist exoskeleton for stroke rehabilitation developed in the Mechatronic and Haptic Interfaces (MAHI) Laboratory at Rice University. The RiceWrist acts on three degrees of freedom (forearm pronation/supination, wrist flexion/ extension, and radial/ulnar deviation), and the MAHI EXOII acts on the same wrist degrees of freedom and one additional degree of freedom (elbow flexion/extension). For performance specifications and differences between the two devices, see $[6,40,41]$.

The MAHI EXO-II currently has three control modes, similar to those originally implemented in the RiceWrist [40]. These control modes are designed for patients with different levels of impairment. For patients who are unable to move the affected limb, there is a passive mode in which the robot carries the patient's joint through a desired trajectory. For patients capable of some voluntary movement and/or force generation, there is a triggered mode that initiates the desired trajectory upon detection of forces over a specified threshold. For patients capable of full movement, there is an active mode in which the robot provides a velocity-dependent resistance against the patient's movement. In the future, the MAHI EXO-II will also include an assist-as-needed mode, which is currently being developed on the RiceWrist [22•]. This controller is based on a modelbased adaptive controller previously developed by Slotine and $\mathrm{Li}$ [96]; this type of controller includes a model of the controlled system (in this case, the patient/robot system) and continually updates estimates of the model parameters while running. In order to assist only as much as needed, the controller is designed to model the residual functional abilities of the subject with position-dependent Gaussian RBFs. The RBFs are defined at many points throughout the workspace, and the parameters related to these functions are estimated during the training by the controller. In order to continually challenge subjects and prevent over-reliance on the robot [57], the adaptive controller is modified so that it possesses uniformly ultimately bounded stability characteristics, rather than asymptotic stability, and a novel feedback modification algorithm is implemented which can modify the allowable error bound and challenge the subjects according to their error performance. Furthermore, in order to avoid applying resistance forces while the subject is "performing better" compared to a previously defined desired trajectory, an online trajectory recalculation algorithm is implemented. The online trajectory recalculation is based on an experimentally defined physiological wrist movement profile, and it generates a position trajectory that is both continuous and time differentiable. The resulting controller is able to assist a patient's movements when the patient lags behind a desired trajectory and allow unimpeded motion if the patient is able to complete the desired trajectory without assistance. This assist-as-needed control algorithm has been tested with 5 healthy subjects and is currently being implemented on MAHI EXO-II and tested with spinal cord injury patients.

Our current work also focuses on promoting active cognitive engagement during therapy by detecting the patient's intent to move using EEG signals. This approach capitalizes on previous work that has demonstrated high classification accuracy of movement intention [97, 98] and builds on that work by incorporating the intention into the control scheme of a therapeutic robot. We make use of the readiness potential, a slow cortical potential that occurs over the central-medial scalp prior to a voluntary movement, as an indicator of the patient's intent to move. The EEG classifier must be calibrated to a specific patient by training a two-class support vector machine to distinguish between move and rest cognitive states. To date, a pilot study has been performed to determine the best mode of operation for the calibration of the EEG classifier [85•, 99]. These modes included passive mode, velocity-triggered mode, backdrive mode (in which the motors are turned off), and observation mode (in which the patient observes another person using the exoskeleton). Three healthy individuals and one stroke subject were used in the experiment. In a one degree-of-freedom target-hitting task with the elbow, significant readiness potential was seen in both the healthy and impaired subjects, and high single- 
trial classification accuracy was achieved. The study showed that the velocity-triggered and passive modes could be viable and pertinent modes of operation for calibrating the EEG system with significantly impaired patients. Our future work includes testing the trained EEG classifier in a closed loop to provide more engaging therapy. It has been hypothesized that the kinesthetic feedback provided during this type of closed loop therapy could enhance presynaptic activity to the cell population or network responsible for moving the impaired limb [100]. Clinical trials will be performed to assess the efficacy of our intention-driven robotic therapy.

\section{Conclusions}

In this review, we have considered the development of robotic systems for upper-limb rehabilitation for stroke patients from a historical perspective and with a focus on modern methods to encourage patient engagement in therapy. The primary benefit of robotic therapy shown so far is the ability to provide highintensity therapy with less effort than traditional therapy, making high-intensity training feasible. The importance of engaging the patient in therapy has been clearly demonstrated through studies of both active and passive movements assisted by robotic systems, and therefore recent research has focused on the ability of robotic systems to engage the patient using assist-as-needed strategies and measures of patient movement intent. These techniques promise to maximize patient effort and engagement by minimizing the level of assistance provided by the robot as much as possible while still enabling subjects to achieve the desired motions. Assist-as-needed strategies have moved toward adaptive controllers that estimate patient effort and impairment in real time based on movement data collected by the robot, thus changing the level of assistance provided even during a single movement. Intention detection using biometrics from EMG and EEG can further involve the patient by providing an online measure of patient effort; these methods may allow inclusion of more severely impaired patients in robotic training protocols. A combination of assist-as-needed methods with online detection of patient intent might further improve patient engagement in therapy.

Although feasibility and positive functional gains have been shown for many of these methods that encourage patient engagement, it is still unclear that which methods are most appropriate for different situations and levels of patient impairment. One might expect that the optimal methods of promoting patient engagement will depend on the movement capabilities of the patient. Assist-as-needed controllers encourage active participation by patients who are able to generate some voluntary movement, but in their current form, they may not be appropriate for severely impaired patients who cannot generate motion because they do not require movement intention if the patient is completely passive. Measures of patient intent also depend on the capabilities of the patient. Triggering movement assistance with a force or velocity threshold is reasonable for patients who are capable of some voluntary force generation or movement, and triggered assistance can enforce a normative movement path. For patients who are unable to generate the necessary force or velocity, EMG and EEG signals can still generate a measure of movement intent to drive the robotic system. EEG allows measure of intent to move in subjects with any level of impairment, but it is currently limited to commanding move and rest signals to single targets (though continuous velocity commands to single targets are starting to become feasible). EMG provides more user control over movements, in some cases allowing users to define their own movement trajectories. However, providing this level of control is only appropriate for users who are able to generate voluntary muscle activation in a normative pattern, since allowing users to define that the trajectory may result in the reinforcement of pathological movements.

Given the many methods available and the strengths and weaknesses of each, it should be possible to design patientspecific training protocols, depending upon each patient's type of injury, level of impairment, and phase of recovery. However, studies completed to date have not explored effects across different patient populations. Carefully controlled, large-scale clinical studies are needed to compare available treatment methods across patient populations and to determine how the efficacy of the methods depends on the characteristics of specific patients. The results of such studies will enable therapists optimize treatment methods for restoring upper-limb function after stroke in patients with a variety of needs and abilities.

Acknowledgments MK O'Malley has consulted for TIRR-Memorial Hermann and received research support from NASA, NSF, NIH and TIRR Foundation.

\section{Compliance with Ethics Guidelines}

Conflict of Interest AA Blank, JA French, and AU Pehlivan all declare no conflicts of interest.

Human and Animal Rights and Informed Consent This article does not contain any studies with human or animal subjects performed by any of the authors.

\section{References}

Papers of particular interest, published recently, have been highlighted as:

- Of importance

•- Of major importance 
1. Go AS, Mozaffarian D, Roger VL, Benjamin EJ, Berry JD, Blaha MJ, Dai S, Ford ES, Fox CS, Franco S, Fullerton HJ, Gillespie C, Hailpern SM, Heit JA, Howard VJ, Huffman MD, Judd SE, Kissela BM, Kittner SJ, Lackland DT, Lichtman JH, Lisabeth LD, Mackey RH, Magid DJ, Marcus GM, Marelli A, Matchar DB, McGuire DK, Mohler ER, Moy CS, Mussolino ME, Neumar RW, Nichol G, Pandey DK, Paynter NP, Reeves MJ, Sorlie PD, Stein J, Towfighi A, Turan TN, Virani SS, Wong ND, Woo D, Turner MB. Heart disease and stroke statistics2014 update: a report from the American Heart Association. Circulation. 2014;129(3):e28-292.

2. Broeks JG, Lankhorst GJ, Rumping K, Prevo AJH. The longterm outcome of arm function after stroke: results of a follow-up study. Disabil Rehabil. 1999;21(8):357-64.

3. Kwakkel G, Wagenaar RC, Koelman TW, Lankhorst GJ, Koetsier JC. Effects of intensity of rehabilitation after stroke a research synthesis. Stroke. 1997;28(8):1550-6.

4. Wing K, Lynskey JV, Bosch PR. Whole-body intensive rehabilitation is feasible and effective in chronic stroke survivors: a retrospective data analysis. Top Stroke Rehabil. 2008;15(3): 247-55.

5. Burgar CG, Lum PS, Shor PC, Van der Loos H. Development of robots for rehabilitation therapy: the Palo Alto VA/Stanford experience. J Rehabil Res Dev. 2000;37(6):663-73.

6. Gupta A, O'Malley MK. Design of a haptic arm exoskeleton for training and rehabilitation. IEEE/ASME Trans Mechatron. 2006;11(3):280-9.

7. Reinkensmeyer DJ, Kahn LE, Averbuch M, McKenna-Cole A, Schmit BD, Rymer WZ. Understanding and treating arm movement impairment after chronic brain injury: progress with the ARM guide. J Rehabil Res Dev. 2000;37(6):653-62.

8. Volpe B, Krebs H, Hogan N, Edelstein L, Diels C, Aisen M. A novel approach to stroke rehabilitation robot-aided sensorimotor stimulation. Neurology. 2000;54(10):1938-44.

9. Williams DJ, Krebs HI, Hogan N. A robot for wrist rehabilitation. In: Proceedings of International Conference of the IEEE Engineering in Medicine and Biology Society (2001). p. 1336-9.

10. Lum P, Burgar CG, Van der Loos M, Shor P, Majmundar M, Yap R. The MIME robotic system for upper-limb neuro-rehabilitation: results from a clinical trial in subacute stroke. In: Proceedings of IEEE International Conference on Rehabilitation Robotics (2005). p. 511-4.

11. Volpe BT, Lynch D, Rykman-Berland A, Ferraro M, Galgano M, Hogan N, Krebs HI. Intensive sensorimotor arm training mediated by therapist or robot improves hemiparesis in patients with chronic stroke. Neurorehabil Neural Repair. 2008;22(3): 305-10.

12. Kahn LE, Lum PS, Rymer WZ, Reinkensmeyer DJ. Robotassisted movement training for the stroke-impaired arm: does it matter what the robot does? J Rehabil Res Dev. 2006;43(5):619-30.

13. Lewis GN, Perreault EJ. An assessment of robot-assisted bimanual movements on upper limb motor coordination following stroke. IEEE Trans Neural Syst Rehabil Eng. 2009;17(6):595-604.

14. Lum PS, Burgar CG, Van der Loos M, Shor PC, Majmundar M, Yap R. MIME robotic device for upper-limb neurorehabilitation in subacute stroke subjects: a follow-up study. J Rehabil Res Dev. 2006;43(5):631-42.

15. Krebs HI, Hogan N. Robotic therapy: the tipping point. Am J Phys Med Rehabil. 2012;91(11 Suppl 3):S290-7.

16. Lynch D, Ferraro M, Krol J, Trudell CM, Christos P, Volpe BT. Continuous passive motion improves shoulder joint integrity following stroke. Clin Rehabil. 2005;19(6):594-9.

17. Hogan N, Krebs HI, Rohrer B, Palazzolo JJ, Dipietro L, Fasoli SE, Stein J, Hughes R, Frontera WR, Lynch D, Volpe BT.
Motions or muscles? Some behavioral factors underlying robotic assistance of motor recovery. J Rehabil Res Dev. 2006;43(5): 605-18.

18. Krebs HI, Volpe B, Hogan N. A working model of stroke recovery from rehabilitation robotics practitioners. J Neuroeng Rehabil. 2009;6:6.

19. Krebs HI, Palazzolo JJ, Dipietro L, Ferraro M, Krol J, Rannekleiv K, Volpe BT, Hogan N. Rehabilitation robotics: performance-based progressive robot-assisted therapy. Auton Robots. 2003;15(1):7-20.

20. Ferraro M, Palazzolo J, Krol J, Krebs H, Hogan N, Volpe B. Robot-aided sensorimotor arm training improves outcome in patients with chronic stroke. Neurology. 2003;61(11):1604-7.

21. Warraich Z, Kleim JA. Neural plasticity: the biological substrate for neurorehabilitation. Phys Med Rehabil. 2010;2(12):S208-19.

22. - Pehlivan AU, Sergi F, O'Malley MK. Adaptive control of a serial-in-parallel robotic rehabilitation device, vol. 2013. In: Proceedings of the IEEE International Conference on Rehabilitation Robotics (2013). A model-based adaptive controller for assist-as-needed control in upper-limb stroke rehabilitation was implemented on the RiceWrist, an exoskeleton for 3-dof wrist rehabilitation. In comparison to a PD controller with fixed gains, the new controller provides comparable tracking performance with lower gains; the more compliant controller should allow subjects to be more independent in completing movements.

23. Pérez-Rodríguez R, Rodríguez C, Costa Ú, Cáceres C, Tormos JM, Medina J, Gómez EJ. Anticipatory assistance-as-needed control algorithm for a multijoint upper limb robotic orthosis in physical neurorehabilitation. Expert Syst Appl. 2014;41(8): 3922-34. Explored the hypothesis that by developing a "dysfunctional" biomechanical model of the patient's unassisted movement, it is possible to predict when the patient will deviate from the prescribed motion path and preemptively apply a corrective force as needed. Normal motions in activities of daily living were recorded from healthy subjects and 5 patients with acquired brain injury. Simulation of an orthosis applying corrective forces showed successful compensation for patients' abnormal movements.

24. - Squeri V, Masia L, Giannoni P, Sandini G, Morasso P. Wrist rehabilitation in chronic stroke patients by means of adaptive, progressive robot-aided therapy. IEEE Trans Neural Syst Rehabil Eng. 2014;22(2):312-25. Subjects completed smallamplitude oscillations with the wrist in one degree of freedom, with assistive torque proportional to the square of the position error. Upon successful completion of a set, the task difficulty was increased by centering the oscillations farther from the neutral position of the joint. Initial trials with 9 stroke subjects showed improved range of motion in the affected joints.

25. Wolbrecht ET, Chan V, Reinkensmeyer DJ, Bobrow JE. Optimizing compliant, model-based robotic assistance to promote neurorehabilitation. IEEE Trans Neural Syst Rehabil Eng. 2008;16(3):286-97.

26. Blank A, O'Malley, MK, Francisco GE, Contreras-Vidal JL. A pre-clinical framework for neural control of a therapeutic upperlimb exoskeleton. In: Proceedings of the IEEE/EMBS International Conference on Neural Engineering (2013). p. 1159-62.

27. Hu X, Tong K, Wei X, Rong W, Susanto E, Ho S. The effects of post-stroke upper-limb training with an electromyography (EMG)-driven hand robot. J Electromyogr Kinesiol. 2013;23(5):1065-74.

28. •- Sarac M, Koyaş E, Erdoğan A, Çetin M, Patoğlu V. Brain computer interface based robotic rehabilitation with online modification of task speed. In: Proceedings of the IEEE International Conference on Rehabilitation Robotics (2013). p. 1-7. Used EEG for online control over the speed of planar arm 
motions. Asked subjects to use motor imagery of the arm moving or resting, and continuously classified a move or rest state. Used the probability of a "move" classification to specify the movement velocity. Demonstrated feasibility with 1 healthy subject.

29. Feil-Seifer D, Mataric MJ. Defining socially assistive robotics. In: Proceedings of IEEE International Conference on Rehabilitation Robotics (2005). p. 465-8.

30. Erlandson RF. Applications of robotic/mechatronic systems in special education, rehabilitation therapy, and vocational training: a paradigm shift. IEEE Trans Rehabil Eng. 1995;3(1):22-34.

31. Charnnarong J, Hogan N, Krebs HI, Sharon A. Interactive robotic therapist. US Patent 5,466,213 (1995).

32. Khalili D, Zomlefer M. An intelligent robotic system for rehabilitation of joints and estimation of body segment parameters. IEEE Trans Biomed Eng. 1988;35(2):138-46.

33. Krebs HI, Hogan N, Aisen ML, Volpe BT. Robot-aided neurorehabilitation. IEEE Trans Rehabil Eng. 1998;6(1):75-87.

34. Lum S, Reinkensmeyer DJ, Lehman SL. Robotic assist devices for bimanual physical therapy: preliminary experiments. IEEE Trans Rehabil Eng. 1993;1(3):185-91.

35. Aisen ML, Krebs HI, Hogan N, McDowell F, Volpe BT. The effect of robot-assisted therapy and rehabilitative training on motor recovery following stroke. Arch Neurol. 1997;54(4):443-6.

36. Lum PS, Burgar CG, Kenney DE, Van der Loos HM. Quantification of force abnormalities during passive and active-assisted upper-limb reaching movements in post-stroke hemiparesis. IEEE Trans Biomed Eng. 1999;46(6):652-62.

37. Charles SK, Krebs HI, Volpe BT, Lynch D, Hogan N. Wrist rehabilitation following stroke: initial clinical results. In: Proceedings of IEEE International Conference on Rehabilitation Robotics (2005). p. 13-6.

38. Loureiro RC, Harwin WS. Reach \& grasp therapy: design and control of a 9-DOF robotic neurorehabilitation system. In: Proceedings of IEEE International Conference on Rehabilitation Robotics (2007). p. 757-63.

39. Masia L, Krebs HI, Cappa P, Hogan N. Design and characterization of hand module for whole-arm rehabilitation following stroke. IEEE/ASME Trans Mechatron. 2007;12(4):399-407.

40. Gupta A, O’Malley MK, Patoglu V, Burgar C. Design, control and performance of RiceWrist: a force feedback wrist exoskeleton for rehabilitation and training. Int $\mathrm{J}$ Robot Res. 2008;27(2):233-51.

41. Pehlivan AU, Celik O, O'Malley MK. Mechanical design of a distal arm exoskeleton for stroke and spinal cord injury rehabilitation. In: Proceedings of IEEE International Conference on Rehabilitation Robotics (2011). p. 1-5.

42. Schabowsky CN, Godfrey SB, Holley RJ, Lum PS. Development and pilot testing of HEXORR: hand EX-oskeleton rehabilitation robot. J Neuroeng Rehabil. 2010;7(1):36.

43. Sledd A, O'Malley MK. Performance enhancement of a haptic arm exoskeleton. In: Proceedings of IEEE Symposium on Haptic Interfaces for Virtual Environment and Teleoperator Systems (2006). p. 375-81.

44. Nef T, Mihelj M, Kiefer G, Perndl C, Muller R, Riener R. ARMin-exoskeleton for arm therapy in stroke patients. In: Proceedings of IEEE International Conference on Rehabilitation Robotics (2007). p. 68-74.

45. Perry JC, Rosen J, Burns S. Upper-limb powered exoskeleton design. IEEE/ASME Trans Mechatron. 2007;12(4):408-17.

46. Sugar TG, He J, Koeneman EJ, Koeneman JB, Herman R, Huang H, Schultz RS, Herring D, Wanberg J, Balasubramanian S, Swenson P, Ward JA. Design and control of RUPERT: a device for robotic upper extremity repetitive therapy. IEEE Trans Neural Syst Rehabil Eng. 2007;15(3):336-46.

47. Lo AC, Guarino PD, Richards LG, Haselkorn JK, Wittenberg GF, Federman DG, Ringer RJ, Wagner TH, Krebs HI, Volpe
BT, Bever CT, Bravata DM, Duncan PW, Corn BH, Maffucci AD, Nadeau SE, Conroy SS, Powell JM, Huang GD, Peduzzi P. Robot-assisted therapy for long-term upper-limb impairment after stroke. N Engl J Med. 2010;362(19):1772-83.

48. Volpe BT, Krebs HI, Hogan N. Is robot-aided sensorimotor training in stroke rehabilitation a realistic option? Curr Opin Neurol. 2001;14(6):745-52.

49. Kahn LE, Zygman ML, Rymer WZ, Reinkensmeyer DJ. Robotassisted reaching exercise promotes arm movement recovery in chronic hemiparetic stroke: a randomized controlled pilot study. J Neuroeng Rehabil. 2006;3:12.

50. Dipietro L, Krebs H, Volpe BT, Stein J, Bever C, Mernoff ST, Fasoli SE, Hogan N. Learning, not adaptation, characterizes stroke motor recovery: evidence from kinematic changes induced by robot-assisted therapy in trained and untrained task in the same workspace. IEEE Trans Neural Syst Rehabil Eng. 2012;20(1):48-57.

51. Pekna M, Pekny M, Nilsson M. Modulation of neural plasticity as a basis for stroke rehabilitation. Stroke. 2012;43(10):2819-28.

52. Adamovich SV, Fluet GG, Tunik E, Merians AS. Sensorimotor training in virtual reality: a review. NeuroRehabilitation. 2009;25(1):29-44.

53. Mihelj M, Nef T, Riener R. A novel paradigm for patientcooperative control of upper-limb rehabilitation robots. Adv Robot. 2007;21(8):843-67.

54. Guidali M, Duschau-Wicke A, Broggi S, Klamroth-Marganska V, Nef T, Riener R. A robotic system to train activities of daily living in a virtual environment. Med Biol Eng Comput. 2011;49(10):1213-23.

55. Tsuji T, Momiki C, Sakaino S (2013) Stiffness control of a pneumatic rehabilitation robot for exercise therapy with multiple stages. In: Proceedings of IEEE/RSJ International Conference on Intelligent Robots and Systems (2013). p. 1480-5.

56. Keller U, Rauter G, Riener R. Assist-as-needed path control for the PASCAL rehabilitation robot. In: Proceedings of IEEE International Conference on Rehabilitation Robotics (2013). p. 1-7.

57. Wolbrecht ET, Chan V, Le V, Cramer SC, Reinkensmeyer DJ, Bobrow JE. Real-time computer modeling of weakness following stroke optimizes robotic assistance for movement therapy, vol. 3. In: Proceedings of International IEEE/EMBS Conference on Neural Engineering (2007). p. 152-8.

58. - Guidali M, Schlink P, Duschau-Wicke A, Riener R. Online learning and adaptation of patient support during ADL training. In: Proceedings of IEEE International Conference on Rehabilitation Robotics (2011). p. 1-6. An adaptive assist-as-needed controller is combined with a dynamic model of the upper limb that is updated in real time. Validation with 7 healthy subjects and 3 stroke subjects showed that the model was able to learn the appropriate amount of assistance to help the subject complete point-to-point reaching motions.

59. Guidali M, Keller U, Klamroth-Marganska V, Nef T, Riener R. Estimating the patient's contribution during robot-assisted therapy. J Rehabil Res Dev. 2013;50(3):379-94.

60. Vergaro E, Casadio M, Squeri V, Giannoni P, Morasso P, Sanguineti V. Self-adaptive robot training of stroke survivors for continuous tracking movements. J Neuroeng Rehabil. 2010;7(13):1-12.

61. Casadio M, Giannoni P, Morasso P, Sanguineti V. A proof of concept study for the integration of robot therapy with physiotherapy in the treatment of stroke patients. Clin Rehabil. 2009;23(3):217-28.

62. Casadio M, Morasso P, Sanguineti V, Giannoni P. Minimally assistive robot training for proprioception enhancement. Exp Brain Res. 2009;194(2):219-31.

63. Papaleo E, Zollo L, Spedaliere L, Guglielmelli E. Patient-tailored adaptive robotic system for upper-limb rehabilitation. In: 
Proceedings of IEEE International Conference on Robotics and Automation (2013). p. 3860-5.

64. Masia L, Casadio M, Giannoni P, Sandini G, Morasso P. Performance adaptive training control strategy for recovering wrist movements in stroke patients: a preliminary, feasibility study. J Neuroeng Rehabil. 2009;6(1):44.

65. $\bullet$ Bower C, Taheri H, Wolbrecht E. Adaptive control with statedependent modeling of patient impairment for robotic movement therapy. In: Proceedings of IEEE International Conference on Rehabilitation Robotics (2013). p. 1-6. Used an adaptive assist-as-needed controller in a two-finger robot designed to assist grasping motions. The controller models inertial and viscous forces, as well as the patient's effort. The effort model includes dependence on position and motion direction. The device was tested with external forces modeling the movement of stroke patients, showing smaller error than previous controllers without direction dependence.

66. Hogan N. Impedance control - an approach to manipulation. ITheory. II-Implementation. III-Applications. J Dyn Syst Meas Control. 1985;107:1-24.

67. Flash T, Hogan N. The coordination of arm movements: an experimentally confirmed mathematical model. J Neurosci. 1985;5(7):1688-703.

68. Krebs H, Volpe B, Williams D, Celestino J, Charles S, Lynch D, Hogan N. Robot-aided neurorehabilitation: a robot for wrist rehabilitation. IEEE Trans Neural Syst Rehabil Eng. 2007;15(3):327-35.

69. Reinkensmeyer DJ, Wolbrecht E, Bobrow J. A computational model of human-robot load sharing during robot-assisted arm movement training after stroke. In: Proceedings of International Conference on IEEE Engineering in Medicine and Biology Society (2007). p. 4019-23.

70. Pérez-Rodríguez R, Rodríguez C, Molina F, Gómez C, Opisso E, Tormos J, Medina J, Gómez E. Simulation-based planification tool for an assistance-as-needed upper limb neurorehabilitation robotic orthosis. In: Proceedings of XIII Mediterranean Conference on Medical and Biological Engineering and Computing (2014). p. 73-6.

71. Rosati G, Bobrow JE, Reinkensmeyer DJ. Compliant control of post-stroke rehabilitation robots: using movement-specific models to improve controller performance. In: Proceedings of ASME International Mechanical Engineering Congress and Exposition (2008). p. 167-74.

72. Squeri V, Basteris A, Sanguineti V. Adaptive regulation of assistance as needed in robot-assisted motor skill learning and neuro-rehabilitation. In: Proceedings of IEEE International Conference on Rehabilitation Robotics (2011). p. 1-6.

73. Sanguineti V, Casadio M, Vergaro E, Squeri V, Giannoni P, Morasso PG. Robot therapy for stroke survivors: proprioceptive training and regulation of assistance. Stud Health Technol Inform. 2009;145:126-42.

74. Badesa FJ, Morales R, Garcia-Aracil N, Sabater J, Casals A, Zollo L. Auto-adaptive robot-aided therapy using machine learning techniques. Comput Methods Programs Biomed. 2013. doi: 10.1016/j.cmpb.2013.09.011

75. Kahn, L., Rymer, W., Reinkensmeyer, D.: Adaptive assistance for guided force training in chronic stroke, vol. 1. In: Proceedings of International Conference of the IEEE on Engineering in Medicine and Biology Society (2004). p. 2722-5.

76. - Novak D, Riener R. Enhancing patient freedom in rehabilitation robotics using gaze-based intention detection. In: Proceedings of IEEE International Conference on Rehabilitation Robotics (2013). p. 1-6. Measured subjects' intent to move with gaze tracking and a velocity threshold. Gaze duration was used for target selection, and robotic assistance was triggered by subject movement over a velocity threshold. Showed feasibility in testing with healthy subjects. Noted a tendency to falsely detect intent to move when a subject was simply examining an object in the environment, and proposed possible solutions to this problem.

77. Lenzi T, De Rossi SMM, Vitiello N, Carrozza MC. Intentionbased EMG control for powered exoskeletons. IEEE Trans Biomed Eng. 2012;59(8):2180-90.

78. - Song R, Tong KY, Hu X, Zhou W. Myoelectrically controlled wrist robot for stroke rehabilitation. J Neuroeng Rehabil. 2013; 10(1):52. Used EMG to detect subject's intent to move. Provided continuous robotic assistance proportional to the normalized EMG readings. Trials with 16 stroke subjects showed improved targeting accuracy.

79. Vaca Benitez, L.M., Tabie, M., Will, N., Schmidt, S., Jordan, M., Kirchner, E.A.: Exoskeleton technology in rehabilitation: towards an EMG-based orthosis system for upper limb neuromotor rehabilitation. J Robot. 2013. doi: 10.1155/2013/610589.

80. Stein J, Narendran K, McBean J, Krebs K, Hughes R. Electromyography-controlled exoskeletal upper-limb-powered orthosis for exercise training after stroke. Am J Phys Med Rehabil. 2007;86(4):255-61.

81. Wang C, Phua KS, Ang KK, Guan C, Zhang H, Lin R, Sui Geok Chua K, Ang BT, Kuah CWK. A feasibility study of noninvasive motor-imagery BCI-based robotic rehabilitation for stroke patients. In: Proceedings of International IEEE/EMBS Conference on Neural Engineering (2009). p. 271-4.

82. - Frisoli A, Loconsole C, Leonardis D, Banno F, Barsotti M, Chisari C, Bergamasco M. A new gaze-BCI-driven control of an upper limb exoskeleton for rehabilitation in real-world tasks. IEEE Trans Syst, Man, Cybern C. 2012;42(6):1169-79. Used EEG combined with gaze tracking to detect subject's intent to move. Gaze tracking and environment information sensed via a Kinect determined the subject's chosen target in a physical environment. EEG signals were continuously classified as "move" or "rest" to trigger movement. Feasibility was demonstrated with 3 healthy subjects and 4 stroke subjects.

83. Ang KK, Guan C, Sui Geok Chua K, Ang BT, Kuah C, Wang C, Phua KS, Chin ZY, Zhang H. Clinical study of neurorehabilitation in stroke using EEG-based motor imagery brain-computer interface with robotic feedback. In: Proceedings of International Conference of the IEEE Engineering in Medicine and Biology Society (2010). p. 5549-52.

84. Gomez-Rodriguez M, Grosse-Wentrup M, Hill J, Gharabaghi A, Scholkopf B, Peters J. Towards brain-robot interfaces in stroke rehabilitation. In: Proceedings of IEEE International Conference on Rehabilitation Robotics (2011). p. 1-6.

85. - Bhagat NA, French JA, Venkatakrishnan A, Yozbatiran N, Francisco GE, O'Malley MK, Contreras-Vidal JL. Detecting movement intent from scalp EEG in a novel upper limb robotic rehabilitation system for stroke. In: Proceedings of International Conference of the IEEE Engineering in Medicine and Biology Society. (2014). Used EEG signals to detect a readiness potential indicating users' intent to move. Calibration testing in a pilot study with 3 healthy subjects and 1 stroke subject.

86. Colombo R, Pisano F, Micera S, Mazzone A, Delconte C, Carrozza MC, Dario P, Minuco G. Robotic techniques for upper limb evaluation and rehabilitation of stroke patients. IEEE Trans Neural Syst Rehabil Eng. 2005;13(3):311-24.

87. Hu XL, Tong KY, Song R, Zheng XJ, Leung WW. A comparison between electromyography-driven robot and passive motion device on wrist rehabilitation for chronic stroke. Neurorehabil Neural Repair. 2009;23(8):837-46.

88. Ho N, Tong K, Hu X, Fung K, Wei X, Rong W, Susanto E. An EMG-driven exoskeleton hand robotic training device on chronic stroke subjects: task training system for stroke rehabilitation. In: Proceedings of IEEE International Conference on Rehabilitation Robotics (2011). p. 1-5. 
89. Tong K, Ho S, Pang P, Hu X, Tam W, Fung K, Wei X, Chen P, Chen M. An intention driven hand functions task training robotic system. In: Proceedings of International Conference on IEEE Engineering in Medicine and Biology Society (2010). p. $3406-9$.

90. Hu X, Tong K, Wei X, Rong W, Susanto E, Ho S. Coordinated upper limb training assisted with an electromyography (EMG)driven hand robot after stroke. In: Proceedings of International Conference on IEEE Engineering in Medicine and Biology Society (2013). p. 5903-6.

91. Venkatakrishnan A, Francisco GE, Contreras-Vidal JL. Applications of brain-machine interface systems in stroke recovery and rehabilitation. Curr Phys Med Rehabil Rep. 2014;2(2): 93-105.

92. Page SJ, Levine P, Leonard A. Mental practice in chronic stroke results of a randomized, placebo-controlled trial. Stroke. 2007;38(4):1293-7.

93. Lotze M, Halsband U. Motor imagery. J Physiol-Paris. 2006; 99(4):386-95.

94. Ang KK, Guan C, Sui Geok Chua K, Ang BT, Kuah C, Wang C, Phua KS, Chin ZY, Zhang H. A clinical study of motor imagerybased brain-computer interface for upper limb robotic rehabilitation. In: Proceedings of International Conference on IEEE Engineering in Medicine and Biology Society (2009). p. $5981-4$.

95. Ang KK, Guan C, Chua KSG, Ang BT, Kuah CWK, Wang C, Phua KS, Chin ZY, Zhang H. A large clinical study on the ability of stroke patients to use an EEG-based motor imagery brain-computer interface. Clin EEG Neurosci. 2011;42(4): 253-8.

96. Slotine J, Li W. On the adaptive control of robot manipulators. Int J Robot Res. 1987;6(3):49-59.

97. Garipelli G, Chavarriaga R, Millán Jdel R. Single-trial analysis of slow cortical potentials: a study on anticipation related potentials. J Neural Eng. 2013;10(3):036014.

98. Lew E, Chavarriaga R, Silvoni S, Millán Jdel R. Detection of self-paced reaching movement intention from EEG signals. Front Neuroeng. 2011;5:13.1-16.

99. French JA. Towards the implementation of non-invasive brain machine interface control on a rehabilitative robotic upper limb exoskeleton. Master's thesis, Rice University; 2014.

100. Dobkin BH. Brain-computer interface technology as a tool to augment plasticity and outcomes for neurological rehabilitation. J Physiol. 2007;579(3):637-42. 